\title{
Effects of FOXM1 inhibition and ionizing radiation on melanoma cells
}

\author{
VIVIENNE S. LEE ${ }^{1 *}$, LUCINDA S. McROBB ${ }^{1 *}$, VAUGHAN MOUTRIE ${ }^{2}$, \\ ESTAVAM D. SANTOS ${ }^{2}$ and TIMOTHY L. SIU ${ }^{1}$ \\ ${ }^{1}$ Department of Clinical Medicine, Faculty of Medicine and Health Sciences, Macquarie University; \\ ${ }^{2}$ Genesis Cancer Care, Macquarie University Hospital, Sydney, New South Wales 2109, Australia
}

Received February 6, 2018; Accepted July 26, 2018

DOI: $10.3892 / 01.2018 .9482$

\begin{abstract}
Metastatic melanoma can be highly refractory to conventional radiotherapy and chemotherapy but combinatorial-targeted therapeutics are showing greater promise on improving treatment efficacy. Previous studies have shown that knockdown of Forkhead box M1 (FOXM1) can sensitize various tumor types to radiation-induced cell death. The effect of combining radiation with a small molecule FOXM1 inhibitor, Siomycin A, on growth, death and migration of a metastatic melanoma cell line (SK-MEL-28) that overexpresses this pleiotropic cell cycle regulator was investigated. Siomycin A (SIOA) was found to be a strong inducer of apoptosis, and inhibitor of proliferation and migration in a scratch wound assay in this cell line. Induction of apoptosis occurred at concentrations $>1 \mu \mathrm{M}$ in association with reductions in the constitutive FOXM1 and anti-apoptotic B-cell lymphoma 2 protein levels found in these cells. Single doses of ionizing radiation (0-40 Gy) delivered by linear accelerator caused inhibition of growth and migration without significant induction of cell death. Pretreatment with SIOA did not increase the sensitivity of this melanoma cell line to radiation as observed in other tumor types. These data confirm that as a single agent, SIOA is an effective inducer of cell death and inhibitor of migration in metastatic melanoma cells expressing constitutive FOXM1. In combination with radiation, SIOA pre-treatment, however, may not be of added benefit.
\end{abstract}

Correspondence to: Dr Timothy L. Siu, Department of Clinical Medicine, Faculty of Medicine and Health Sciences, Macquarie University, Suite 201, 2 Technology Place, Sydney, New South Wales 2109, Australia

E-mail: tsiu@mqneurosurgery.com

*Contributed equally

Key words: FOXM1, irradiation, melanoma, thiazole antibiotics, Siomycin A

\section{Introduction}

Melanoma is one of the deadliest forms of cancer known to humans and has the highest propensity to metastasize to the brain. Current therapeutics for metastatic brain tumours include surgery and radiation therapy but despite technological advancement on improving treatment safety and efficacy with these modalities, brain metastases remain a major cause of death in patients with metastatic melanoma $(1,2)$.

In search of more effective therapeutics, an oncogenic signalling pathway driven by the transcription factor Forkhead box M1 (FOXM1) has emerged as a promising anti-metastatic target in recent years. FOXM1 protein belongs to a class of highly evolutionary conserved mammalian transcription factors which underlie the regulation of many fundamental homeostatic and developmental processes $(3,4)$. In normal cells, FOXM1 serves as a key regulator of cell cycle progression and cellular development, mediating G1-S and G2-M phase transition and maintaining a balance between cell proliferation and apoptosis in developing cells (4). While its expression is turned off in terminally differentiated cells, an aberrant gain of FOXM1 function has been shown to link to tumorigenesis $(3,4)$. Emerging evidence has demonstrated that FOXM1 is upregulated in a multitude of solid tumours including breast, lung, basal cell, pancreatic, hepatocellular, ovarian and prostate carcinomas, rendering it one of the most overexpressed genes in human cancers $(5,6)$. More importantly, recent studies have indicated that the oncogenic mechanisms of FOXM1 involve not only cell cycling dysregulation as predicted from its physiological function but the control of a wide array of oncogenic pathways and events including inhibition of cell differentiation, apoptosis and DNA repair and promotion of cell invasion, migration and angiogenesis, quintessential of the expression of the metastatic phenotypes (4). Conceivably targeting FOXMI could result in an across-the-board inhibition of many of the pro-metastatic events and combining with existing ablative treatment with focused irradiation, highly selective and localized cancer cell death can potentially be induced with low toxicity to normal tissue.

Recently several lines of evidence have lent support to the roles of FOXM1 expression in inducing metastatic melanoma and suppression of FOXM1 via RNA interference, blocking 
peptides, or chemotherapeutic agents has proven successful in countering tumorigenesis (7-10). Importantly new evidence has indicated that combined treatment of FOXM1 inhibition with a small molecule inhibitor Siomycin A (SIOA) and irradiation can achieve a higher rate of cell death in glioblastoma cell lines, thus raising the possibility of harnessing FOXMI inhibitors as a radiosensitizing agent (11). Currently there is a dearth of information in the role of FOXM1 inhibition in sensitizing radioresistant melanoma and we therefore sought to elucidate the cellular effects of irradiation and SIOA on metastatic melanoma cells and determine the effects of pretreatment with SIOA on cellular response to irradiation.

\section{Materials and methods}

Cell culture and irradiation. A panel of ATCC-derived (SK-MEL-28, A375) or cultured, short-term patient-derived (WMD009, WMD046, MM200, SMU027) melanoma cells were kindly provided by Professor H. Rizos (Macquarie University, Sydney, Australia) to identify a FOXM1 overexpressing strain. All cell lines were previously published except for WMD046 which is a short term patient derived sample sourced from Professor Rizos (12-16). Cells were maintained in Dulbecco's Modified Eagle's medium (DMEM; Gibco; Thermo Fisher Scientific, Inc., Waltham, MA, USA) with $10 \%$ fetal bovine serum (FBS), $20 \mathrm{mM}$ HEPES and $4 \mathrm{mM}$ L-glutamine and cultured at $37^{\circ} \mathrm{C}$ in humidified air with $5 \%$ carbon dioxide. Cells were routinely passaged at $80 \%$ confluence with $0.1 \%$ Trypsin/EDTA (Sigma-Aldrich; Merck KGaA, Darmstadt, Germany). Cells were seeded in 8-well chamber slides, 96- or 6-well plates for irradiation with X-rays (0-40 Gy) generated by a $6 \mathrm{MV}$ linear accelerator (LINAC; Elekta Synergy, Crawley, UK) at Macquarie University Hospital (Sydney, Australia) or for SIOA treatment. SIOA was purchased from Sigma-Aldrich; Merck KGaA and dissolved in dimethyl sulfoxide (DMSO; Sigma-Aldrich; Merck KGaA). DMSO constituted a final concentration of $0.1 \%$ in all assays.

Trypan blue viability assay. Viable-to-dead cell ratios were determined 1-5 days after drug or radiation treatment using the trypan blue viability assay. Briefly, floating and adherent cells were collected, washed and stained with trypan blue for $10 \mathrm{~min}$ before automated counting of white (live) or blue (permeable, dead) cells with an automated cell counter (Countess II FL Automated Cell Counter; Thermo Fisher Scientific, Inc.).

MTT proliferation assay. Cells were seeded in 96-well plates at $5 \times 10^{3}$ cells per well in $5 \%$ serum-containing medium and allowed to adhere overnight before treatment with drug or radiation. Treatment proceeded for $24,48,72$ or $120 \mathrm{~h}$. At least 8 replicate wells were used for each dose and time point within each independent experiment. Five $\mathrm{h}$ prior to the end of each incubation period, $20 \mu 1$ of MTT (Thermo Fisher Scientific, Inc.) was added to each well $(0.5 \mathrm{mg} / \mathrm{ml})$ and the plates incubated at $37^{\circ} \mathrm{C}$ for a further $5 \mathrm{~h}$. The medium was then discarded by inversion and the cells resuspended in $200 \mu 1$ of DMSO per well to dissolve the formazan product. The plates were mechanically shaken for 5-10 min and the absorbance read at 560/670 $\mathrm{nm}$ within $1 \mathrm{~h}$ using a microplate reader (BMG Labtech PHERAstar FS; Thermo Fisher Scientific, Inc,).

Scratch wounding cell migration assay. A scratch wound assay was used to examine cellular migration after SIOA and radiation treatment. Cells were seeded in 8 -well chamber slides (Nunc; Thermo Fisher Scientific, Inc.) or 6-well culture plates to obtain $90-100 \%$ confluence. Cells were irradiated with single doses of radiation (0-40 Gy) or SIOA $(0-5 \mu \mathrm{M})$ for $1 \mathrm{~h}$ prior to scratch wounding of the cell layer with a $1 \mathrm{ml}$ pipette tip. The medium was replaced with or without the thiazole antibiotic to remove floating cells and debris. The scratch wound area was immediately imaged with an upright inverted microscope and reimaged at 24,48 and $72 \mathrm{~h}$ post-wounding. When sequential combination treatments were given, a period of $24 \mathrm{~h}$ was given before addition of the second treatment.

Images were analyzed using Image J (Rasband, W.S., ImageJ, National Institutes of Health, Bethesda, Maryland, USA; https://imagej.nih.gov/ij/, 1997-2016). For every scratch wound, a series of 4 images were taken along the length of the wounded area and measured by blinded observer. For each image, a region of interest (ROI; rectangle) was drawn that aligned with the upper and lower edges of the image and the observed division between cell regrowth and denudation. The area within the ROI was recorded for each image and divided by the area measured immediately after scratch wounding on day 0 to give wound area as a percentage of the original scratch wound area. These wound area percentages for each dose and time point from 3 independent experiments were then averaged $(n=3)$ and plotted.

Western blot analysis. Protein lysates were prepared in immunoprecipitation buffer $(50 \mathrm{mM}$ Tris- $\mathrm{HCl}, \mathrm{pH} 7.5$, $150 \mathrm{mM}$ sodium chloride, $0.5 \%$ deoxycholate, $0.1 \%$ sodium dodecyl sulphate, 1\% NP40 substitute, 5 mM EDTA) supplemented with freshly prepared protease inhibitor cocktail (GE Healthcare, Chicago, IL, USA). Protein concentration was determined using the BCA protein assay (Pierce Biotechnology Inc.; Thermo Fisher Scientific, Inc.). Whole cell protein extracts $(30 \mu \mathrm{g})$ were resolved by SDS-PAGE, transferred to a PVDF membrane using the iblot transfer system (Thermo Fisher Scientific, Inc.) and probed with primary antibodies, species-specific HRP-conjugated secondary antibodies and detected by enhanced chemiluminescence. The following antibodies were used: Anti-FOXM1 (H-19 sc-501 rabbit polyclonal); anti-FOXM1 (G-5 sc-376471 mouse monoclonal; both Santa Cruz Biotechnology, Inc., Dallas, TX, USA); anti-cleaved poly (ADP-ribose) polymerase 1 (PARP1; ab2321 rabbit polyclonal; Abcam, Cambridge, UK); anti-B-cell lymphoma 2 ( $\mathrm{Bcl} 2$; sc-492 rabbit polyclonal, Santa Cruz Biotechnology, Inc.); anti-phospho-AKT (Ser473) (cs-4060S rabbit monoclonal; Cell Signalling Biotechnology, Inc., Danvers, MA, USA); anti-superoxide dismutase 2 (Sod2; ab13534 rabbit polyclonal; Abcam). All membranes were probed with antibodies targeting either $\beta$-actin (A5060.5 rabbit polyclonal; Sigma-Aldrich; Merck KGaA) or GAPDH (ab181602 rabbit monoclonal; Abcam) as protein loading controls. 


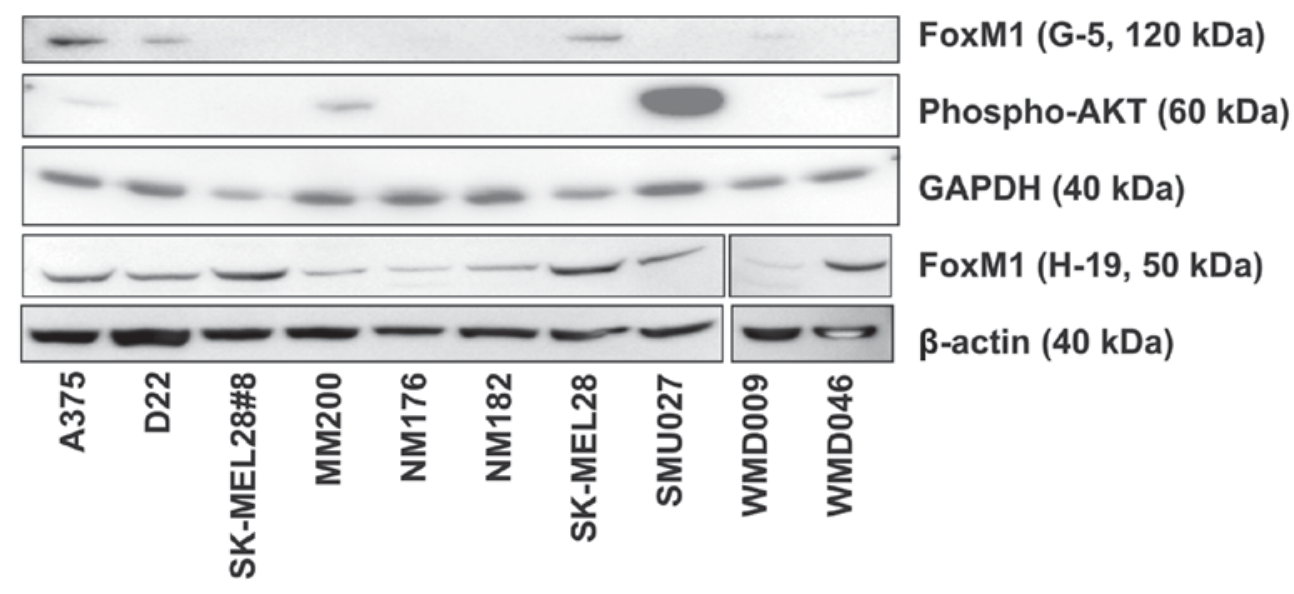

Figure 1. FoxM1 expression is variable in melanoma cell lines. Whole cell protein lysates were prepared from cultured melanoma cells as listed. Proteins ( $30 \mu \mathrm{g})$ were separated by SDS-PAGE prior to western transfer to PVDF membrane. Membranes were probed with antibodies targeting FOXM1 and phospho-AKT (Ser473). HRP-tagged secondary antibodies were detected with enhanced chemiluminescence. Antibodies targeting GAPDH or $\beta$-actin were used to determine equal loading. MELRMU8 (SK-MEL-28\#8) is a subclone of original ATCC strain. FOXM1, Forkhead box M1.

Statistical analysis. Data are presented as mean \pm standard error of the mean (SEM). Effects of single treatments over time were analyzed by two-way ANOVA with Dunnett's post-hoc analysis for multiple group comparisons using Prism 6.04 software (Graphpad Software, Inc., La Jolla, CA, USA). P<0.05 was considered to indicate a statistically significant difference.

\section{Results}

Constitutive FOXM1 expression in melanoma cell lines. FOXM1 expression has been noted to be constitutively activated in many cancers including melanoma and to contribute to tumorigenesis and chemo- and radio-resistance (5,7-9). A series of 10 melanoma cell lines were tested for FOXM1 protein expression by Western blotting with two different FOXM1 antibodies. A mouse monoclonal anti-FOXM1 antibody (G-5) recognized a single band of approximately $120 \mathrm{kDa}$, consistent with the full length FOXM1 protein, in strains A375, D22, SK-MEL-28 and faintly in WMD009 (Fig. 1). In contrast, a polyclonal rabbit anti-FoxM1 antibody (H-19) identified a primary band on western blots at approximately $50 \mathrm{kDa}$ with a faint band at $80 \mathrm{kDa}$ (Fig. 1). The pattern of expression of the $80 \mathrm{kDa}$ band in strains A375, D22 and SK-MEL-28 was consistent with that of the $120 \mathrm{kDa}$ band (G-5). The $50 \mathrm{kDa}$ band was expressed in all cell lines but was considered non-specific as it did not respond to SIOA treatment. In view of these, the H-19 mouse monoclonal antibody was selected for further studies.

As constitutive activation of the AKT pathway can influence FOXM1 expression (8), we also examined the relative levels of expression of phosphorylated AKT in the 10 melanoma cell lines. Expression of phosphorylated AKT (Ser473) was observed only in A375, MM200, SMU027 and WMD046 (Fig. 1). There appeared to be no correlation between the relative expression levels of FOXM1 and phospho-AKT. Collectively the SK-MEL-28 cell line was chosen for further studies given its high expression levels of FOXM1.

SIOA inhibits SK-MEL-28 proliferation and induces cellular apoptosis. SK-MEL-28 cells were treated with SIOA at concentrations of $0-10 \mu \mathrm{M}$ for up to 5 days. Cell proliferation and death were measured using trypan blue viability assays (Fig. 2A and B) and MTT proliferation assays (Fig. 2C). Vehicle controls (0.1\% DMSO) continued to grow over the $72 \mathrm{~h}$ period. At a dose of $0.6 \mu \mathrm{M}$ SIOA, cell growth was inhibited 1.5 -fold by $72 \mathrm{~h}(\mathrm{P}<0.0001)$ while doses $>1.2 \mu \mathrm{M}$ completely inhibited growth and significantly increased the proportion of dead cells in the first $48 \mathrm{~h}$ period. The $\mathrm{IC}_{50}$ was determined as $0.67 \mu \mathrm{M}$ (Fig. 2D).

SIOA inhibits SK-MEL-28 cell migration. Cellular migration was examined in a scratch wound assay. At concentrations $>1.2 \mu \mathrm{M}$, SIOA primarily induced cell death across the entire plate of cells (see phase-contrast images, Fig. 2E). At sub-lethal concentrations $(\leq 0.6 \mu \mathrm{M})$, cellular migration was inhibited without significant cell death after 3 days (Fig. 2E and F).

SIOA decreases FOXM1 and BCL2 protein expression in SK-MEL-28 melanoma cells with a concomitant increase in PARP-1 cleavage. Protein expression of FOXM1 and two FOXM1-regulated genes, the anti-oxidant enzyme, SOD2, and the anti-apoptotic protein, BCL2, were examined in response to SIOA (Fig. 3). FOXM1 (full length protein, $120 \mathrm{kDa}$ ) was abruptly reduced at concentrations between 1.2 and $2.5 \mu \mathrm{M}$ SIOA. Levels of SOD2 increased dose-dependently, however BCL2 decreased between 1.2 and $2.5 \mu \mathrm{M}$ SIOA, in line with the decreases in FOXM1 expression observed. Expression of the p65 subunit of the NFKB transcription factor decreased in a linear fashion with SIOA dose. The late apoptotic marker, cleaved PARP1, was evident at concentrations above $1.2 \mu \mathrm{M}$ SIOA up to $48 \mathrm{~h}$, consistent with the level of cell death observed.

Radiation inhibits SK-MEL-28 growth with minimal effects on cell death. Total cell counts were determined over a period of 5 days for SK-MEL-28 melanoma cells exposed to radiation doses up to $40 \mathrm{~Gy}$. Cells continued to grow in the absence of irradiation up to day 5 until growth reached $100 \%$ confluence, while radiation inhibited cell growth at all doses (Fig. 4A and B). In the irradiated cells, cell numbers increased between day 1 and day 3 at all doses, but declined moderately between days 3 

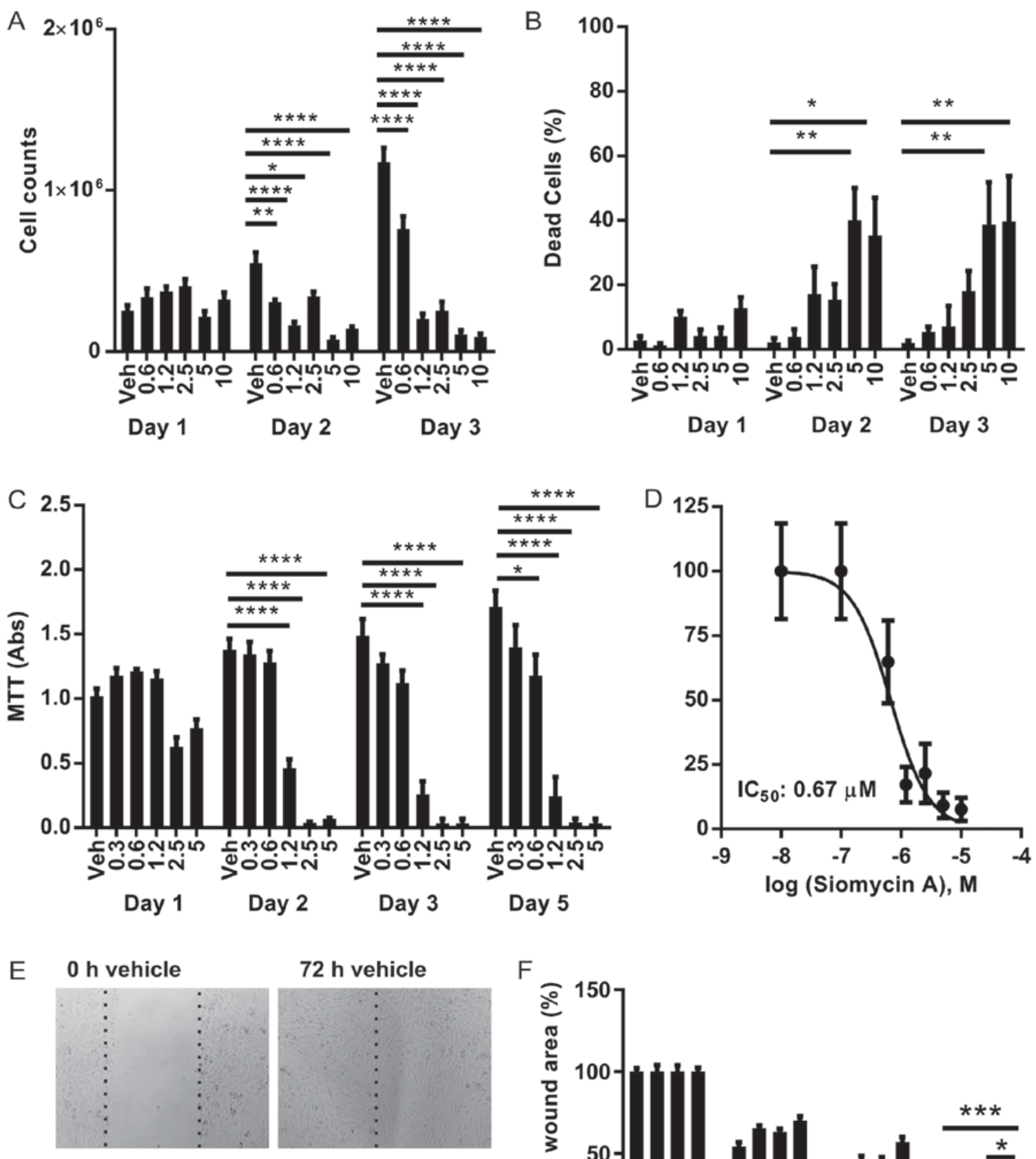

$72 \mathrm{~h}$ vehicle
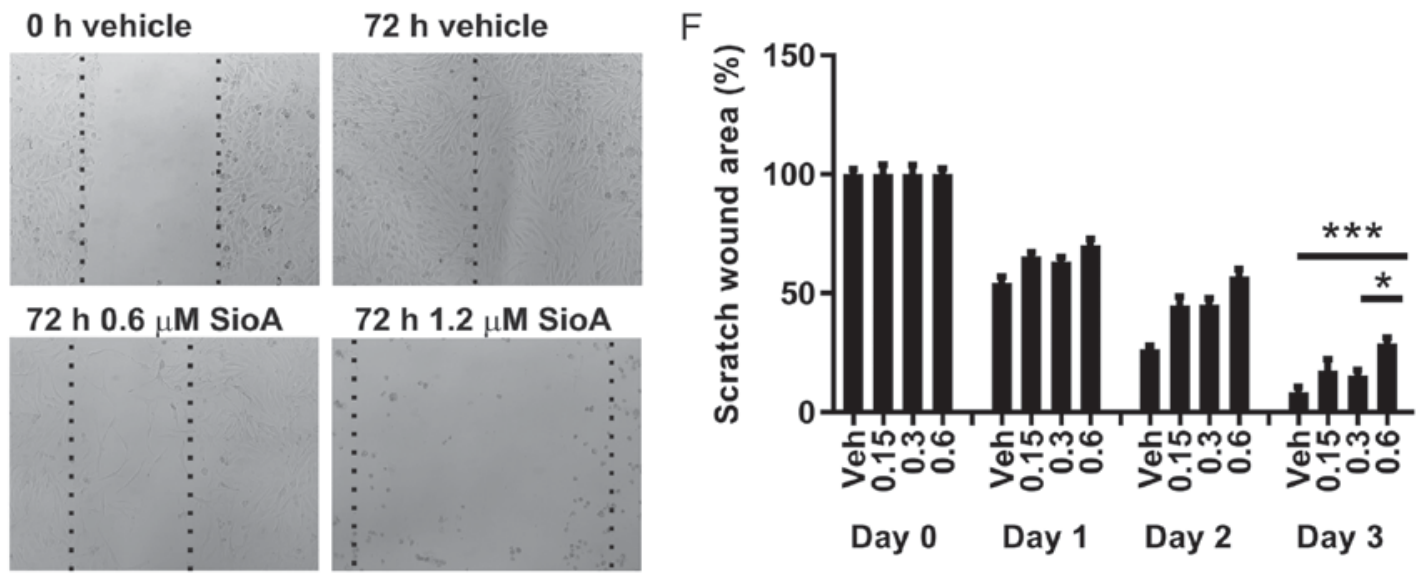

Day 0 Day 1 Day 2 Day 3

Figure 2. SIOA induces growth inhibition and cell death in SK-MEL-28 melanoma cells. SK-MEL-28 cells were treated with SIOA at doses of 0-10 $\mu$ M and monitored for viability and migration for up to 5 days. (A) Cell counts (trypan blue-negative cells/ml) by trypan blue assay and automated cell counting. Data shown as mean \pm SEM and representative of 2 independent experiments $(n=2)$; (B) Proportion of dead cells ( $\%$ of total) measured by trypan blue viability assay and automated cell counter. Data shown as mean \pm SEM and representative of 2 independent experiments ( $\mathrm{n}=2)$; (C) Cell proliferation was examined by MTT assay. Mean \pm SEM of 3 independent assays $(n=3)$; (D) Dose-response curve for SIOA in SK-MEL-28; (E) Representative images of wound area after treatment at day 0 and day 3. Dotted lines approximate the borders between cellular and denuded areas. Original magnification, x50. (F) Wound area (\% of vehicle control at time zero) after scratch injury in response to SIOA treatment. Data represent mean \pm SEM of 3 independent experiments (n=3). Two-way ANOVA with Dunnett's post-hoc test: ${ }^{*} \mathrm{P}<0.05,{ }^{* *} \mathrm{P}<0.01,{ }^{* * *} \mathrm{P}<0.001,{ }^{* * * *} \mathrm{P}<0.0001$. SEM, standard error of the mean; SIOA, Siomycin A.

and 5 at the higher doses (10 and $20 \mathrm{~Gy}$ ). The percentage of dead, trypan blue-positive cells did not change significantly over the time period in response to radiation at all doses and did not reach $>8 \%$ at any time (Fig. 4 C), consistent with the inability to detect the apoptotic marker, cleaved PARP-1, on western blots (not shown). An MTT proliferation assay confirmed the predominance of growth inhibition rather than cell death at radiation doses of 10-40 Gy (Fig. 4D). 


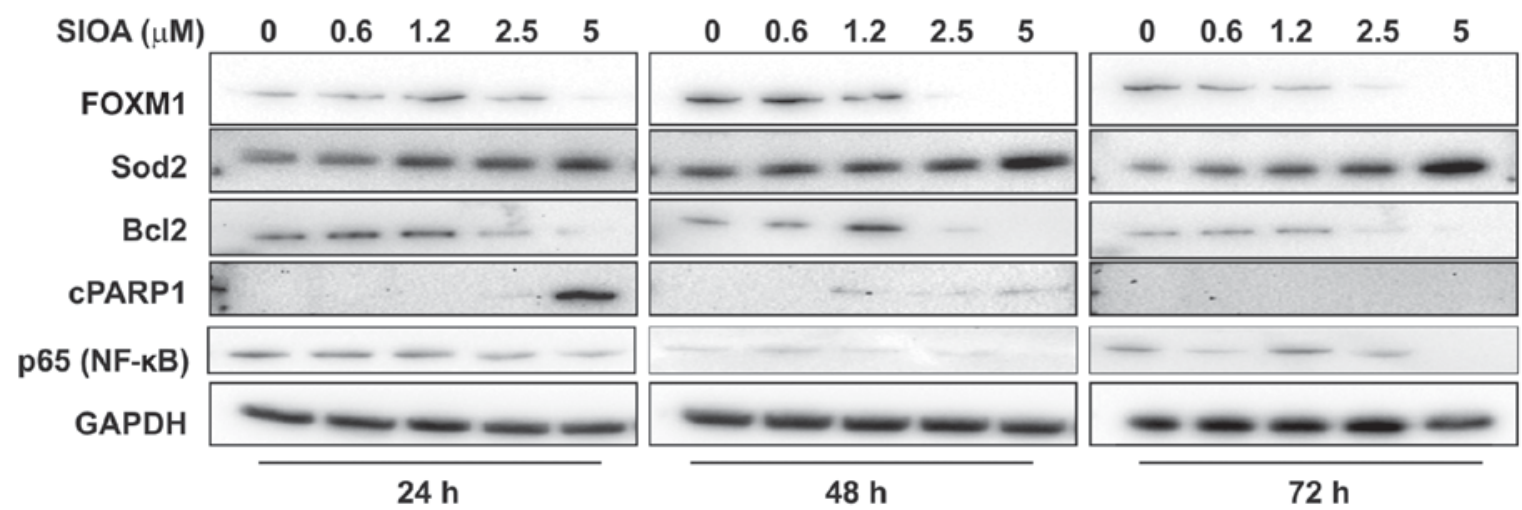

Figure 3. SIOA inhibits FoxM1 expression in SK-MEL-28 melanoma cells. SIOA (0.6-5 $\mu \mathrm{M})$ or vehicle (0.1\% DMSO) was added to SK-MEL-28 cells and total protein harvested after 24, 48 and $72 \mathrm{~h}$. Western analysis was performed with antibodies targeting FoxM1 (120 kDA), Sod2 (25 kDa), Bcl2 (26 kDa), cleaved PARP1 (85 kDa), p65 (NFkB) $(65 \mathrm{kDa})$, and GAPDH (40 kDa). Blots are representative of 3 independent experiments. SIOA, Siomycin A; DMSO, dimethyl sulfoxide; FOXM1, Forkhead box M1; Sod2, superoxide dismutase 2; Bcl2, B-cell lymphoma 2; PARP-1, polymerase 1.

Radiation inhibits SK-MEL-28 migration in a scratch wound assay. Wound area after scratch injury was measured consecutively in live cells over 3 days in response to radiation. Wound area (as a percentage of denuded area measured immediately after scratch injury) continued to decline at all doses over the time period as cells repopulated the denuded area (Fig. 4E and F). Inhibition of regrowth was seen at all doses however this reached significance at doses of $20 \mathrm{~Gy}(\mathrm{P}<0.05)$ and 40 Gy $(\mathrm{P}<0.01)$.

Radiation does not alter FOXM1 expression in SK-MEL-28 melanoma cells. No significant change in FOXM1 protein expression was observed in SK-MEL-28 cells in response to radiation (Fig. 5). SOD2 and BCL2 protein expression increased transiently with radiation dose at the $24 \mathrm{~h}$ time point relative to the non-irradiated control. BCL2 expression was moderately reduced at the higher doses (20 and 40 Gy) at later time points, however SOD2 expression was not different to controls.

Pretreatment with SIOA at sub-lethal doses has no radio-sensitizing effect on $S K-M E L-28$ melanoma cells. To examine whether radiation and SIOA could act synergistically on inhibition of SK-MEL-28 cell growth and migration, cells were treated with a mid-range dose of radiation (10 Gy) combined with SIOA. Cells were treated with Siomycin A $24 \mathrm{~h}$ prior to radiation treatment to allow FoxM1 knockdown prior to irradiation. As earlier experiments showed complete cell loss after 2 days with concentrations of SIOA $>1.2 \mu \mathrm{M}$, cells were examined at sub-lethal concentrations of 0.3 and $0.6 \mu \mathrm{M}$ SIOA with 10-40 Gy doses of radiation.

MTT proliferation assays were performed to establish any synergistic effects of SIOA and radiation on cell proliferation. At a dose of $0.3 \mu \mathrm{M}$ (Fig. 6A), Siomycin A had minimal effect on proliferation in these assays, while at $0.6 \mu \mathrm{M}$, SIOA significantly reduced proliferation from day 3 onwards (Fig. 6B), consistent with earlier results (Fig. 2). In combination with radiation, no further reduction in proliferation was observed over the time period at either dose (Fig. 6A and B). Similarly, no synergistic effects of SIOA and radiation were observed on migration (Fig. 6C and D). At high doses $(>1 \mu \mathrm{M})$, apoptosis was absolute within 2 days in the 8 -well chamber slides and no further contribution from radiation could be observed. At sub-lethal concentrations (0.3-0.6 $\mu \mathrm{M})$, there were no synergistic effects on migration at radiation doses of 10 and 20 Gy measured at $72 \mathrm{~h}$ after radiation (Fig. 6C and D).

\section{Discussion}

Overexpression of the pleiotropic transcription factor FOXM1 has been observed in many human malignancies and regulates multiple pathways important for tumorigenesis $(5,6)$. Recent studies demonstrate FOXM1 overexpression in malignant melanoma and an association with cancer stage and prognosis suggesting FOXM1 knockdown may be a novel therapeutic target (7-9). Consistent with these studies, we observed reduction in FOXM1 at micromolar Siomycin A doses in the metastatic melanoma cell line, SK-MEL-28, with a rather rapid drop-off in FOXMI expression at concentrations above $1 \mu \mathrm{M}$. BCL2 was similarly inhibited by SIOA in the same dose range and this is of particular importance as BCL2 is a well-known anti-apoptotic factor and a downstream target of FOXM1 (17). BCL2 and FOXM1 inhibition occurred simultaneously with an increase in expression of the apoptotic marker, cleaved PARP-1. BCL2 is also a target of the NFKB transcription factor (18), and downregulation may have been associated with the simultaneous decrease observed in constitutive $N F \kappa B(R E L A / P 65)$ expression. In other studies, proteasome inhibitor-induced apoptosis is at least partly mediated via suppression of the $\mathrm{NF} \kappa \mathrm{B}$ signalling pathway (19). Our results illustrated a rapid induction of apoptosis at doses between 1-2 $\mu \mathrm{M}$ Siomycin A and are in agreement with the regulatory role of FOXM1 and BCL2.

SOD2 has been demonstrated to be both a FOXM1 $(20,21)$ and $\mathrm{NF}_{\mathrm{K}} \mathrm{B}$ (22) transcriptional target but our data suggested an uncoupling of this relationship in response to Siomycin A-we found that $S O D 2$ expression was unchanged despite a dramatic loss of $F O X M 1$ and $N F \kappa B$ expression. In addition we observed a transient induction of $S O D 2$ in response to radiation but this did not correlate with FOXM1 expression, which remained unchanged.SOD2 is an important scavenger enzyme controlling intracellular reactive oxygen species (ROS), and overexpression is a known contributor to both chemo- and radioresistance in various tumor types (23). Silencing FOXM1 was shown to 


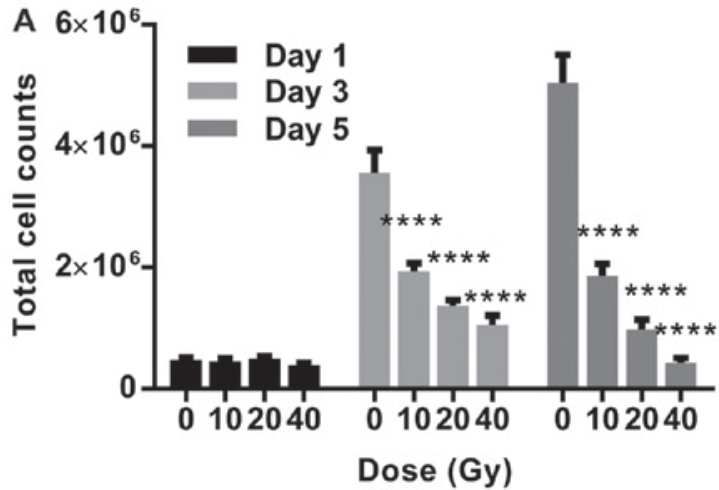

B

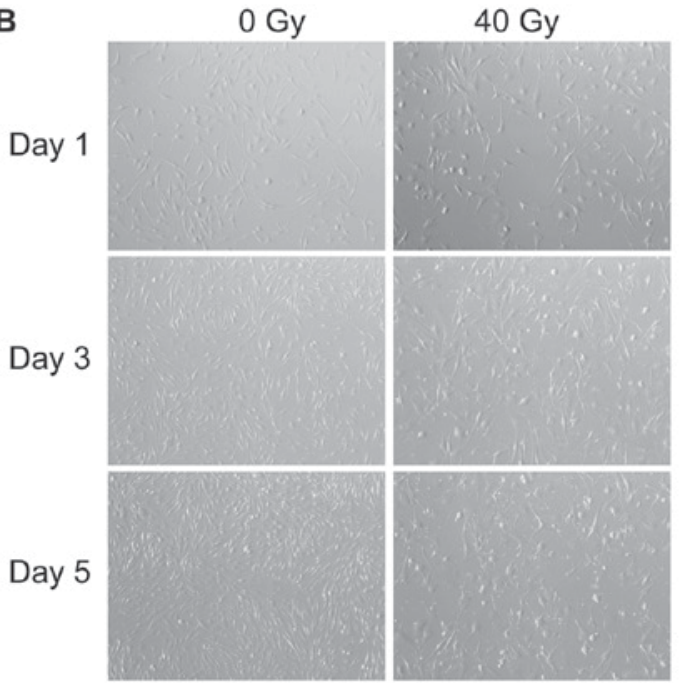

C

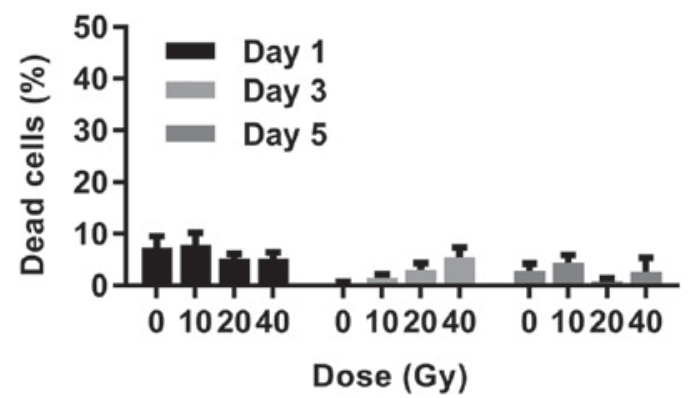

D
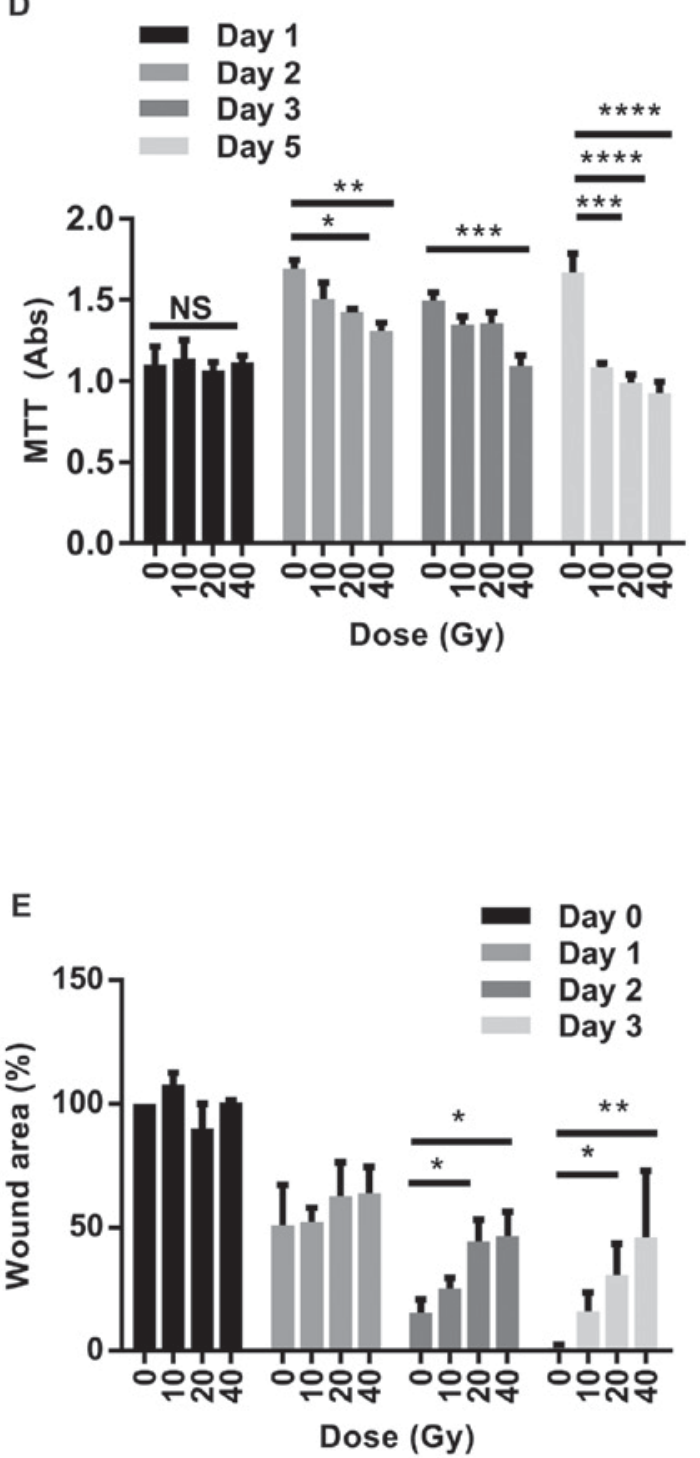

F

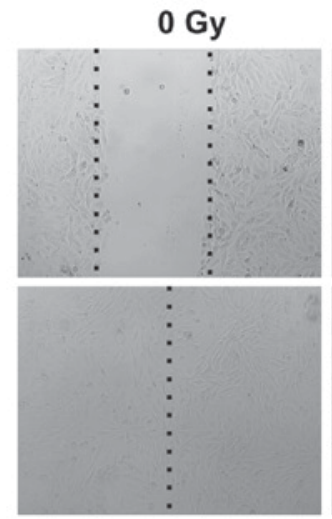

$10 \mathrm{~Gy}$

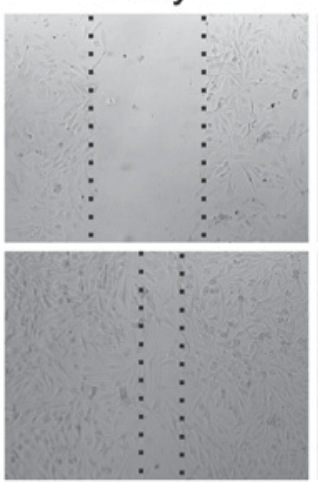

20 Gy

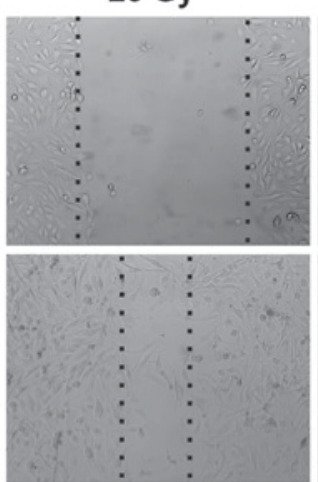

40 Gy

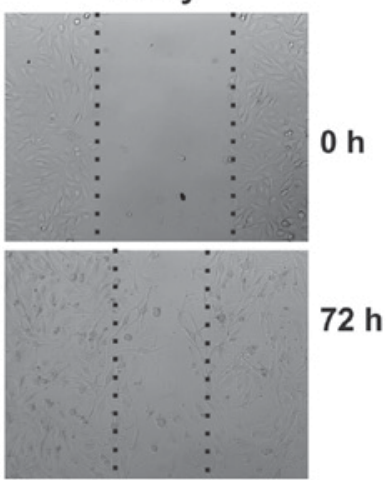

Figure 4. Radiation inhibits growth and migration of SK-MEL-28 melanoma cells without significant cell death. SK-MEL-28 cells were irradiated at doses of 0-40 Gy by linear accelerator and monitored for viability and migration for up to 5 days. (A) Cell counts (trypan blue-negative cells/ml) by trypan blue assay and automated cell counting. Data shown as mean \pm SEM and representative of 2 independent experiments; (B) Representative images of SK-MEL-28 cell morphology after irradiation. Original magnification, 25x; (C) Proportion of dead cells (\% of total) measured by trypan blue viability assay and automated cell counter. Data shown as mean \pm SEM and is representative of 2 independent experiments. (D) Cell proliferation measured by MTT assay, mean \pm SEM of 3 independent experiments; (E) Wound area after scratch injury in response to radiation at 0-40 Gy dose. Data represent mean \pm SEM of 3 independent experiments; (F) Representative images of wound area after irradiation at day 0 and day 3 . Dotted lines approximate the borders between cellular and denuded areas. Original magnification, x50. Two-way ANOVA with Dunnett's post-hoc test: ${ }^{*} \mathrm{P}<0.05,{ }^{* * *} \mathrm{P}<0.01,{ }^{* * *} \mathrm{P}<0.001,{ }^{* * * *} \mathrm{P}<0.0001$. SEM, standard error of the mean. 


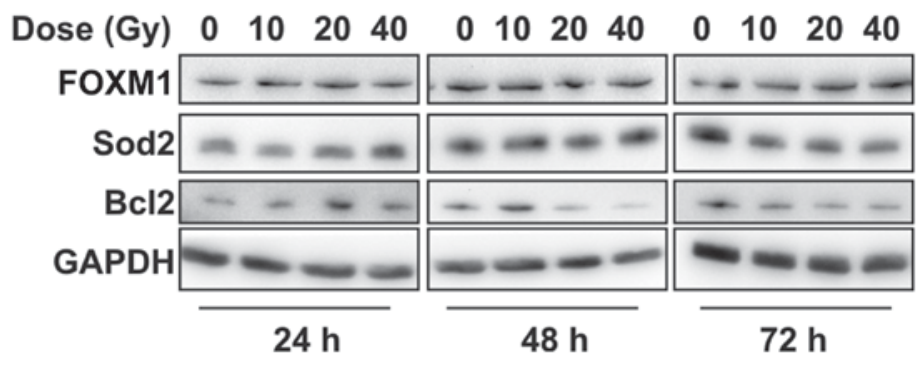

Figure 5. Radiation has no inhibitory effect on FoxM1 in SK-MEL-28 melanoma cells. Doses of 0-40 Gy X-ray radiation were delivered to SK-MEL-28 cells in 6-well plates and total protein harvested after 24, 48 and $72 \mathrm{~h}$. Western analysis was performed with antibodies targeting FoxM1 (120 kDA), Sod2 (25 kDa), Bcl2 $(26 \mathrm{kDa})$, and GAPDH (44 kDa). Cleaved PARP-1 was not detected at any time point (not shown). Blots are representative of 2 independent experiments FOXM1, Forkhead box M1; Sod2, superoxide dismutase 2; Bc12, B-cell lymphoma 2.
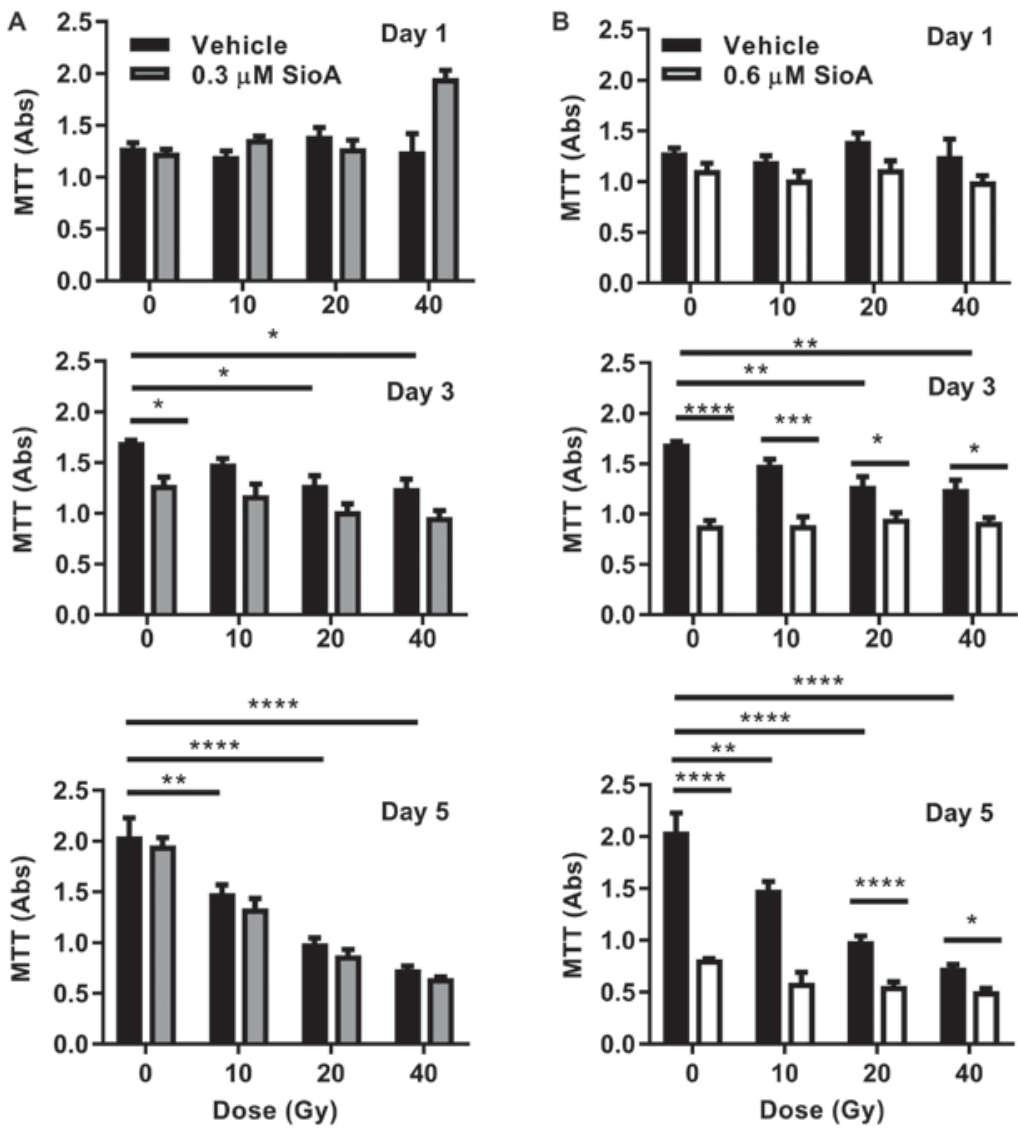

C

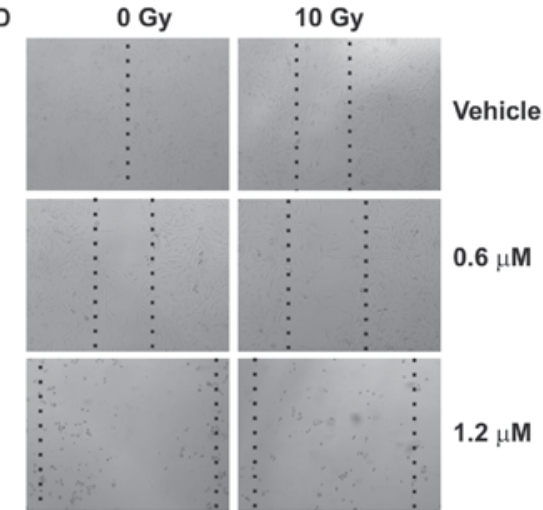

Figure 6. Combinatorial effects of SIOA and radiation on cell proliferation and migration. Cells were treated with SIOA $24 \mathrm{~h}$ prior to irradiation by linear accelerator. Cell proliferation was monitored at day 1,3 and 5 post-irradiation by MTT assay with $0.3 \mu \mathrm{m}$ (A) or $0.6 \mu \mathrm{M}$ (B) SIOA. Data represent mean \pm SEM of 3 independent experiments. (C) Cell migration was monitored at day 3 post-irradiation with 0,10 and 20 Gy doses. Mean \pm SEM of 3 independent experiments. (D) Representative images of scratch wound areas $72 \mathrm{~h}$ after irradiation at $0-10 \mathrm{~Gy}$ after pretreatment with 0.6 or $1.2 \mu \mathrm{M}$ SIOA. Dotted lines approximate the borders between cellular and denuded areas. Original magnification, $x 50$. Two-way ANOVA with Dunnett's post-hoc test: ${ }^{*} \mathrm{P}<0.05,{ }^{* * *} \mathrm{P}<0.01,{ }^{* * * *} \mathrm{P}<0.001$, ${ }_{* * * * *} \mathrm{P}<0.0001$. SIOA, Siomycin A; SEM, standard error of the mean. 
decrease SOD2 expression, increase intracellular ROS levels and in turn the sensitivity to ROS inducers (not radiation), enhancing apoptosis in vitro and in vivo (21). We think that the observed FOXM1-SOD2 uncoupling may have contributed to the inability of SIOA to sensitize melanoma cells to radiation. In line with this observation, others have shown that radiosensitivity can be enhanced by downregulation of $N F \kappa B$ and SOD2 (24) and increased chemosensitivity can be induced by simultaneous BCL2 and SOD2 knockdown in resistant melanoma (25). Collectively further studies on FOXM1-SOD2 interactions in tumor cells with different radiosensitivity would shed light on the underpinnings of radioresistance.

To our knowledge, this is the first study to examine the effects of combining SIOA as a FOXM1 inhibitor with radiation in melanoma cells. Combination of FOXM1 suppression and radiation was previously investigated in various tumor cell lines, which overall suggested a potential synergistic treatment effect $(11,17,26)$. Our results contrast with these findings, however, in that while the basal expression of FOXM1 was significantly induced by radiation in various non-melanoma cancer cells types, radiation appeared to have no effect on FOXM1 expression. Similarly, robust apoptosis was induced by radiation in various non-melanoma cells but only cell stasis was observed here, even up to doses of $40 \mathrm{~Gy}$. This dose range is well above that used in other combination studies and valid in the clinical setting, hence the radiation dose range does not appear a limiting factor in this study $(11,17,26)$. Rather, it appears that the basal level of sensitivity of individual cell types to radiation and the ability to further induce FOXM1 may predict the efficacy of combination treatment. Such notion of cell specificity is echoed by a lymphoma study in which synergistic induction of apoptosis in several cell lines treated with combined proteasome inhibitor and radiation was noted to be dependent on the p53 status with mutant or null p53 cells exhibiting a lack of sensitisation and vice versa (27). On this note, the observed resistance to radiosensitization of SK-MEL-28, being p53 mutant (28), corroborates with these findings.

It should be noted that in this study we used the MTT assay as a surrogate for the use of clonogenic survival assays. Good correlation has been demonstrated between clonogenic survival and MTT assays, especially when multiple and extended time points are examined to account for the acute and post-acute phases of the radiation response (29). However it should be made clear that our findings of lack of synergism refer to the properties of proliferation and migration and relationships to clonal survival and invasion are inferred from the important role they play in these phenotypes.

We demonstrated that FOXM1-overexpressing melanoma cells, SK-MEL-28, were highly susceptible to SIOA-induced apoptosis which in turn was associated with FOXM1, BCL2 and $\mathrm{NF}_{\kappa} \mathrm{B}$ inhibition. SIOA treatment, however, did not further sensitize these radioresistant cells to combined radiation treatment. The combination of FOXM1 inhibition and radiation therapy may be ineffective for radioresistant melanoma.

\section{Acknowledgements}

The authors would like to thank Professor H. Rizos (Macquarie University) for supplying the melanoma strains used in the present study.

\section{Funding}

The present study was supported by a research grant provided by Brain Foundation, Australia (grant no. 9201200696).

\section{Availability of data and materials}

The datasets used and/or analyzed during the current study are available from the corresponding author on reasonable request.

\section{Authors' contributions}

VSL was involved in data acquisition (all techniques), analysis and interpretation of data, manuscript drafting and revision, and the final approval of the manuscript. LSM was involved in the conception and design of the study (methodology), data acquisition (migration, western blotting), analysis and interpretation of data, manuscript drafting and revision, and the final approval of the manuscript. VM and EDS were involved in the conception and design of the study (radiation), manuscript revision, and the final approval of the manuscript. TLS was involved in the conception and design of the whole study, analysis and interpretation of data, manuscript drafting and revision, the final approval of the manuscript. All authors read and approved the final manuscript.

\section{Ethics approval and consent to participate}

Not applicable.

\section{Patient consent for publication}

Not applicable.

\section{Competing interests}

The authors declare that they have no competing interests.

\section{References}

1. Khan MK, Khan N, Almasan A and Macklis R: Future of radiation therapy for malignant melanoma in an era of newer, more effective biological agents. Onco Targets Ther 4: 137-148, 2011.

2. Bullard DE, Cox EB and Seigler HF: Central nervous system metastases in malignant melanoma. Neurosurgery 8: 26-30, 1981.

3. Myatt SS and Lam EW: The emerging roles of forkhead box (Fox) proteins in cancer. Nat Rev Cancer 7: 847-859, 2007.

4. Wang Z, Ahmad A, Li Y, Banerjee S, Kong D and Sarkar FH: Forkhead box M1 transcription factor: A novel target for cancer therapy. Cancer Treat Rev 36: 151-156, 2010.

5. Xu XS, Miao RC, Wan Y, Zhang LQ, Qu K and Liu C: FoxM1 as a novel therapeutic target for cancer drug therapy. Asian Pac J Cancer Prev 16: 23-29, 2015.

6. Wierstra I: FOXM1 (Forkhead box M1) in tumorigenesis: overexpression in human cancer, implication in tumorigenesis, oncogenic functions, tumor-suppressive properties, and target of anticancer therapy. Adv Cancer Res 119: 191-419, 2013.

7. Ito T, Kohashi K, Yamada Y, Maekawa A, Kuda M, Furue M and Oda Y: Prognostic significance of forkhead box M1 (FoxM1) expression and antitumour effect of FoxM1 inhibition in melanoma. Histopathology 69: 63-71, 2016.

8. Miyashita A, Fukushima S, Nakahara S, Yamashita J, Tokuzumi A, Aoi J, Ichihara A, Kanemaru H, Jinnin M and Ihn H: Investigation of FOXM1 as a potential new target for melanoma. PLoS One 10: e0144241, 2015. 
9. Kruiswijk F, Hasenfuss SC, Sivapatham R, Baar MP, Putavet D, Naipal KA, van den Broek NJ, Kruit W, van der Spek PJ, van Gent DC, et al: Targeted inhibition of metastatic melanoma through interference with Pin1-FOXM1 signaling. Oncogene 35: 2166-2177, 2016.

10. Bhat UG, Zipfel PA, Tyler DS and Gartel AL: Novel anticancer compounds induce apoptosis in melanoma cells. Cell Cycle 7: $1851-1855,2008$

11. Maachani UB, Shankavaram U, Kramp T, Tofilon PJ, Camphausen K and Tandle AT: FOXM1 and STAT3 interaction confers radioresistance in glioblastoma cells. Oncotarget 7: 77365-77377, 2016.

12. Carlino MS, Todd JR, Gowrishankar K, Mijatov B, Pupo GM, Fung C, Snoyman S, Hersey P, Long GV, Kefford RF and Rizos H: Differential activity of MEK and ERK inhibitors in BRAF inhibitor resistant melanoma. Mol Oncol 8: 544-554, 2014.

13. Carlino MS, Gowrishankar K, Saunders CA, Pupo GM, Snoyman S, Zhang XD, Saw R, Becker TM, Kefford RF, Long GV and Rizos H: Antiproliferative effects of continued mitogen-activated protein kinase pathway inhibition following acquired resistance to BRAF and/or MEK inhibition in melanoma. Mol Cancer Ther 12: 1332-1342, 2013.

14. Long GV, Fung C, Menzies AM, Pupo GM, Carlino MS, Hyman J, Shahheydari H, Tembe V, Thompson JF, Saw RP, et al: Increased MAPK reactivation in early resistance to dabrafenib/trametinib combination therapy of BRAF-mutant metastatic melanoma. Nat Commun 5: 5694, 2014

15. Zhang XD, Franco A, Myers K, Gray C, Nguyen T and Hersey P: Relation of TNF-related apoptosis-inducing ligand (TRAIL) receptor and FLICE-inhibitory protein expression to TRAIL-induced apoptosis of melanoma. Cancer Res 59: 2747-2753, 1999

16. Pupo GM, Boyd SC, Fung C, Carlino MS, Menzies AM, Pedersen B, Johansson P, Hayward NK, Kefford RF, Scolyer RA, et al: Clinical significance of intronic variants in BRAF inhibitor resistant melanomas with altered BRAF transcript splicing. Biomark Res 5: 17, 2017.

17. Halasi M and Gartel AL: Suppression of FOXM1 sensitizes human cancer cells to cell death induced by DNA-damage. PLoS One 7: e31761, 2012.

18. Fahy BN, Schlieman MG, Mortenson MM, Virudachalam S and Bold RJ: Targeting BCL-2 overexpression in various human malignancies through NF-kappaB inhibition by the proteasome inhibitor bortezomib. Cancer Chemother Pharmacol 56: 46-54, 2005 .
19. Almond JB and Cohen GM: The proteasome: A novel target for cancer chemotherapy. Leukemia 16: 433-443, 2002.

20. Park HJ, Carr JR, Wang Z, Nogueira V, Hay N, Tyner AL, Lau LF, Costa RH and Raychaudhuri P: FoxM1, a critical regulator of oxidative stress during oncogenesis. EMBO J 28: 2908-2918, 2009.

21. Halasi M, Pandit B, Wang M, Nogueira V, Hay N and Gartel AL: Combination of oxidative stress and FOXM1 inhibitors induces apoptosis in cancer cells and inhibits xenograft tumor growth. Am J Pathol 183: 257-265, 2013.

22. Kinugasa H, Whelan KA, Tanaka K, Natsuizaka M, Long A, Guo A, Chang S, Kagawa S, Srinivasan S, Guha M, et al: Mitochondrial SOD2 regulates epithelial-mesenchymal transition and cell populations defined by differential CD44 expression. Oncogene 34: 5229-5239, 2015.

23. Hosoki A, Yonekura S, Zhao QL, Wei ZL, Takasaki I, Tabuchi Y, Wang LL, Hasuike S, Nomura T, Tachibana A, et al: Mitochondria-targeted superoxide dismutase (SOD2) regulates radiation resistance and radiation stress response in HeLa cells. J Radiat Res 53: 58-71, 2012.

24. Sun Y, St Clair DK, Fang F, Warren GW, Rangnekar VM, Crooks PA and St Clair WH: The radiosensitization effect of parthenolide in prostate cancer cells is mediated by nuclear factor-kappaB inhibition and enhanced by the presence of PTEN. Mol Cancer Ther 6: 2477-2486, 2007.

25. Benlloch M, Mena S, Ferrer P, Obrador E, Asensi M, Pellicer JA, Carretero J, Ortega A and Estrela JM: Bcl-2 and Mn-SOD antisense oligodeoxynucleotides and a glutamine-enriched diet facilitate elimination of highly resistant B16 melanoma cells by tumor necrosis factor-alpha and chemotherapy. J Biol Chem 281: 69-79, 2006.

26. Lee Y, Kim KH, Kim DG, Cho HJ, Kim Y, Rheey J, Shin K, Seo YJ, Choi YS, Lee JI, et al: FoxM1 promotes stemness and radio-resistance of glioblastoma by regulating the master stem cell regulator Sox2. PLoS One 10: e0137703, 2015.

27. Kurland JF and Meyn RE: Protease inhibitors restore radiation-induced apoptosis to Bcl-2-expressing lymphoma cells. Int J Cancer 96: 327-333, 2001.

28. Avery-Kiejda KA, Zhang XD, Adams LJ, Scott RJ, Vojtesek B, Lane DP and Hersey P: Small molecular weight variants of p53 are expressed in human melanoma cells and are induced by the DNA-damaging agent cisplatin. Clin Cancer Res 14: 1659-1668, 2008.

29. Buch K, Peters T, Nawroth T, Sänger M, Schmidberger H and Langguth P: Determination of cell survival after irradiation via clonogenic assay versus multiple MTT Assay-a comparative study. Radiat Oncol 7: 1, 2012. 